\title{
La eficacia de un modelo de instrucción directa \\ en la enseñanza de la comprensión de ideas principales*
}

\section{James F. Baumann}

Universidad Purdue

Traducción: Silvia Sánchez-Herrero

Las revisiones del alcance de las series de libros de lectura básicos y de los gráficos de resultados, los libros de texto de métodos de lectura y las pruebas de comprensión lectora, dan testimonio del hecho de que enseñar y examinar la comprensión de las ideas principales es una práctica - educativa muy extendida (Armbruster, Stevens y Rosenshine, 1977; Chou Hare y Miligan, en prensa; Jenkins y Pany, 1980; Johnson y Barrett, 1981; National Assessment of Educational Progress, 1981; Otto y Askov, 1974; Rosenshine, 1980a; Winograd y Brennan, 1983). Intuitivamente, la comprensión de las ideas principales es una destreza lectora importante: cuando los lectores se enfrentan a un texto, no pueden recordarlo entero $y$ es conveniente que puedan discriminar entre las ideas más y menos importantes para retener así en la memoria, de un modo eficaz, la información esencial de un texto. Por lo tanto, el conocimiento clásico ha relacionado intimamente la aptitud para identificar y recordar las ideas principales o los elementos centrales de una historia, con una buena comprensión lectora.

De hecho, la destreza en la comprensión de la información importante de un texto en prosa, discrimina los buenos lectores de los deficientes. (Eamon, 1978-79; Smiley, Oakley, Worthen, Campione y Brown, 1977; Winograd, 1984).
Además, Winograd ha señalado que la sensibilidad hacia lo importante explica una proporción significativa de varianza en varias medidas de comprensión general en estudiantes del grado 8, incluso cuando ya se habían tenido en cuenta las diferencias en el CI y en la aptitud para decodificar. En otras palabras, hay datos empíricos que apoyan la noción de que la aptitud para comprender las ideas principales, no sólo discrimina entre los lectores deficientes y los buenos, sino que también está relacionada con medidas más globales de la comprensión.

Aunque muchos estudios han indicado que los adultos lectores fluidos, son capaces de comprender lo esencial, el tema, las ideas principales, o centrales de un texto en prosa (Duchastel, 1979; Johnson, 1970; Kintsch y Keenan, 1973; Kintsch, Kozminsky, Streby, McKoon y Keenan, 1975; Meyer, 1975; Meyer y McConkie, 1973), las investigaciones llevadas a cabo con niños son contradictorias. Mientras que algunos estudios sugieren que los lectores jóvenes tienen bastante destreza a la hora de comprender las ideas principales (ver la revisión de Baker y Stein, 1981; Brown y Smiley, 1977; Christie y Schumacher, 1975; Danner, 1976; Meyer, 1977; Walters, 1978), otros indican que los niños tienen dificultades importantes en esta tarea (Baumann, 1981, 1983a; Dunn, Matthews y

* "The effectiveness of a direct instruction paradigm for training main idea comprehension" Reading Researcb Quarterly, 1984, XX, 1, 93-115. Reproducido con autorizació. (C) de la traducción, infancia $y$ aprendizaje. 
Bieger, 1979; Otto, Barrett y Koenke, 1968; Taylor, 1980; Tierney, Bridge y Cera, 1978-79; Winograd, 1984). En una serie de experimentos, Williams y sus colaboradores (Taylor y Williams, 1983; Williams, Taylor y deCani, en prensa; Williams, Taylor y Ganger, 1981) investigaron la aptitud de niños y adultos para identificar las ideas principales de un texto. Williams encontró que la comprensión de la idea principal no era una destreza que simplemente los lectores poseian o no; más bien, se observaron algunas diferencias evolutivas significativas y algunas diferencias en la ejecución de la tarea: los lectores mayores tendían a aumentar la destreza global en la comprensión de la idea principal, pero el tipo de tarea utilizado para determinar la comprensión de las ideas principales (por ej., elegir el mejor título, escribir una frase-resumen, identificar las frases anómalas) también afectaba la ejecución. Baumann (1982) se dio cuenta sin embargo, de que muchos de los estudios que sugerían que los niños eran diestros en la comprensión de las ideas principales, poseian niveles bajos de validez externa (ecológica); por ejemplo, los ambientes en los que se realizaban las investigaciones eran dramáticamente distintos de los escenarios típicos de la escuela en los que los niños leían y comprendian textos de prosa escritos. Por lo tanto, dada la fuerte relación que hay entre la sensibilidad hacia lo importante y la comprensión, unido a la dificultad que tienen los niños para entender las ideas principales cuando leen textos reales en el ambiente escolar, se deduce que los alumnos necesitan una enseñanza eficaz de esta destreza.

Durante la última década, se han averiguado muchas cosas sobre los procesos cognitivos y psicológicos subyacentes implicados en la comprensión lectora (McConkie, 1978; Moore y Readence, 1983; Pearson, 1984a; Reder, 1978; Spiro, Bruce y Brewer, 1980), pero hasta hace poco los investigadores no habian estudiado el tema de cómo enseñar de un modo eficaz las destrezas en la comprensión lectora (Guthrie, 1981; Pearson, 1984a; Santa y Hayes, 1981; Tierney y Cunningham, 1984). Aunque no hay muchas investigaciones sobre la enseñanza de la comprensión (ver la revisión de Pearson, 1982, 1984b; Pearson y Gallag- her, 1983), los resultados de los pocos estudios existentes han sido alentadores. De la cantidad de trabajos realizados sobre la eficacia del profesor (Berliner, 1981; Berliner y Rosenshine, 1977; Brophy, 1979; Brophy y Good, en prensa; Duffy, 1981, 1982; Good y Brophy, 1984; Fisher, Berliner, Filby, Marliave, Cahen y Dishaw, 1980; Rosenshine, 1980b; Rosenshine y Berliner, 1978; Rosenshine y Stevens, 1984), los estudios de enseñanza de la comprensión que emplean los principios de la «instrucción directa» (Berliner, 1981; Duffy y Roehler, 1982) han demostrado que puede enseñarse a los niños unas destrezas específicas en la comprensión. Por ejemplo, Hansen (1981) y Hansen y Pearson (1983) obtuvieron resultados positivos al enseñar a los niños a aumentar de forma sensible la destreza en la comprensión; Raphael y Pearson (1982) y Raphael y McKinney (1983) señalaron los efectos positivos del entrenamiento metacognitivo sobre las estrategias de los niños al preguntar o al responder; Reis y Spekman (1983) tuvieron éxito en el entrenamiento de los lectores deficientes de grado superior en el uso de estrategias de control de la comprensión; Fitzgerald y Spiegel (1983) aumentaron la comprensión de historias en algunos niños, mediante la instrucción directa sobre la estructura narrativa; Tharp (1982) señaló los niveles superiores de ejecución en la comprensión lectora en un estudio longitudinal que implicaba a algunos niños polinesio-hawaianos de primaria, niños de alto riesgo educativo, que recibieron una instrucción activa y concentrada de la comprensión, cuando se les comparó con otros niños a los que se les aplicó un programa más tradicional centrado en la decodificación; Patching, Kameenui, Carnine, Gersten y Colvin (1983) entrenaron a estudiantes del grado 5 en las destrezas críticas de la comprensión lectora (generalización defectuosa, causalidad incorrecta y testimonios inválidos) y encontraron que los estudiantes que recibían una instrucción directa y sistemática, rendían mejor que otros estudiantes comparables, que recibieron una enseñanza de feedback-correctivo-con-librode trabajo y unos controles. Tal y como Pearson (1982) concluye después de revisar muchos de estos estudios: «los datos son alentadores. Parece como si después 
de todo, pudiésemos enseñar las destrezas de la comprensión» (p. 22).

Varios autores han recomendado estrategias y métodos para enseñar la comprensión de la idea principal (Alexander, 1976; Aulls, 1978; Axelrod, 1975; Dishner y Readence, 1977; Donlan, 1980; Putnam, 1974; Santeusanio, 1983; Taylor, 1978), pero ninguna de estas técnicas está basada en la investigación actual sobre la instrucción eficaz en la comprensión y ninguna se ha probado empíricamente. El único estudio sobre instrucción realizado hasta la fecha, es uno de Williams (1984) en el que a estudiantes con problemas de aprendizaje de una escuela media, se les enseñó a comprender las ideas principales de párrafos cortos. Los resultados indican que el grupo de instrucción rendía significativamente mejor que el grupo control en una tarea post-test de escribir un resumen. Sin embargo, generalizar estos resultados a la instrucción de las ideas principales en las clases de las escuelas corrientes, sería inapropiado, debido a la población usada en este estudio y al ámbito bastante limitado de las ideas principales (simples párrafos descriptivos).

A la luz del éxito que los investigadores han tenido al entrenar a estudiantes en las destrezas en la comprensión cuando la instrucción había sido sistemática y directa, el propósito de este estudio era desarrollar un modelo de instrucción directa para enseñar a los niños la comprensión de las ideas principales y evaluar su relativa eficacia frente a la instrucción tradicional (libros de lectura básicos) sobre las ideas principales, y sobre la no instrucción.

Aunque el término instrucción directa no tiene una definición universalmente aceptada (ver Berliner, 1981; Duffy y Roehler, 1982), para el propósito de este estudio se adoptó la propuesta por Duffy y Roehler (1982), ya que incluía todos los principios de la investigación acerca de la eficacia del profesor sobre los que se basaba la estrategia de instrucción (Baumann, 1983b). Para Duffy y Roehler, la «instrucción directa significa un tema didáctico central, una secuenciación precisa de contenido, una alta implicación del alumno, un cuidadoso control del profesor y un feedback correctivo específico a los estudiantes» (p. 35).
Pero, como ha señalado Baumann (1983b), el verdadero motor del modelo de instrucción directa, es el profesor:

en la instrucción directa, el profesor, de una manera razonablemente formal y cara a cara, dice, muestra, construye modelos, demuestra, enseña la destreza que hay que aprender. La palabra clave es maestro puesto que es quien está al mando de la situación de aprendizaje y quien dirige la lección, en comparación con tener la instrucción «dirigida» por un libro, un centro de aprendizaje, un cuadernillo o por un conjunto de materiales (p. 287)

Por lo tanto, el modelo de instrucción directa usado en este estudio, requirió que el maestro fuera el responsable del tema didáctico central de la enseñanza, de la secuenciación de los contenidos, de la implicación del alumno, del control y del feedback correctivo, con un cambio gradual de la responsabilidad del aprendizaje del profesor al estudiante a medida que progresaban las lecciones (Pearson y Gallagher, 1983).

En este estudio, que se llevó a cabo con alumnos del grado 6 , se administró al grupo experimental (grupo de Estrategia) una serie de lecciones sobre las ideas principales que se ajustaban al modelo de instrucción directa desarrollado por Baumann (1983b), en el que a cada lección seguía un proceso de cinco pasos: (a) introducir la destreza (Instrucción), (b) ofrecer un ejemplo (Ejemplo), (c) enseñar directamente la destreza (Instrucción Directa), (d) proporcionar la aplicación y ejercicios de transferencia bajo la supervisión del profesor para ofrecer el feedback correctivo (aplicación Dirigida por el Profesor) y (e) administrar ejercicios prácticos (Práctica independiente). Puesto que los programas de lectura básicos han sido criticados debido a la sustitución de los maestros por unas cuantas lecciones que de hecho enseñan (dan una instrucción directa en) las destrezas en la comprensión (Durkin, 1981; Mason, 1983), a un segundo grupo experimental (grupo Básico) se le administró una serie de lecciones sobre las ideas principales y sobre detalles complementarios tomadas directamente de libros básicos de lectura, actualmente en curso (Clymer y Venezky, 1982). El grupo Control dedicó una cantidad equivalente 
del tiempo de instrucción en actividades no relacionadas con el lenguaje. Los resultados de este estudio indican que. la aplicación del modelo de instrucción directa es muy eficaz para enseñar a niños del grado 6 a comprender las ideas principales en un texto de prosa escrito.

\section{METODO}

\section{Sujetos y Diseño}

Participaron en el estudio 66 estudiantes del grado 6 de una escuela primaria de un pueblo rural del medio oeste. Todos los alumnos formaban parte de un grupo del grado 6 con tres profesores. que utilizaban un ambiente de clase físicamente abierto. Todos los estudiantes del grado 6 tomaron parte en el estudio, con la excepción de un niño con problemas de aprendizaje y de otro alumno que no obtuvo el permiso de sus padres para realizar el estudio.

Se empleó un diseño postest $3 \times 3$, donde el tratamiento (Estrategia, Básico y Control) se cruzaba con el nivel de rendimiento (Alto, Bajo, Medio). Para determinar este nivel, se pasó el nivel de comprensión lectora del Metropolitan Achievement Test (Prescott, Balow, Hogan y Farr, 1978) en su forma JS intermedia. Los bloques del nivel de rendimiento se forman con 22 estudiantes asignados al azar a cada tratamiento de la siguiente manera: de los 21 estudiantes que mejor puntuaron en el Metropolitan (bloque superior, puntuación media de 847, que correspondia a un percentil 92) se asignaron al azar 7 de ellos a cada tratamiento. Del mismo modo, de los 24 estudiantes que obtuvieron las puntuaciones medias (bloque medio, puntuación media de 770 y un percentil de 66) se asignaron a cada tratamiento 8 al azar; $\mathrm{y}$ de los 21 estudiantes con las puntuaciones más bajas (bloque inferior, puntuación media de 713 y percentil 38) se escogieron de forma aleatoria a 7 en cada una de las condiciones experimentales. Asi, cada grupo estaba formado por 7 estudiantes de rendimiento alto, $8 \mathrm{del}$ medio y 7 del bajo.

\section{Procedimiento}

Tanto los dos grupos experimentales (Estrategia y Básico) como el grupo Control, recibieron 8 lecciones de $30 \mathrm{mi}$ nutos cada una, con toda la instrucción dada por el experimentador (uno de los maestros de la enseñanza primaria). Las clases se distribuyeron en un período de 2,5 semanas y se contrabalancearon de forma que en un día cualquiera sólo se encontraran dos de los tres grupos. Toda la instrucción tenía lugar durante el período normal dedicado a la lectura $y$, cuando los estudiantes no se encontraban en el grupo-tratamiento con el experimentador, estaban con uno de los profesores de su clase dedicados al aprendizaje de la lectura (los maestros de la clase asignaron a todos los estudiantes unos "contratos» de lectura puesto que no estaban en sus grupos habituales de lectura). El postest, que también se llevó a cabo en el periodo de lectura, tuvo lugar dos dias después del final de la instrucción. Se intentó hacer el experimento de una forma lo más natural posible y los estudiantes aceptaron fácilmente al experimentador como un profesor más del grupo.

\section{Grupos Experimentales}

Grupo de Estrategia. El contenido de las lecciones para el grupo de Estrategia consistia en enseñar a los estudiantes una jerarquía de destrezas de las ideas principales que iba desde encontrar las ideas principales explícitas e implícitas en párrafos, a encontrar las ideas principales explícitas e implícitas en textos cortos y a hacer un resumen de las ideas principales de pasajes cortos. La tabla 1 muestra una breve descripción de cada una de las ocho lecciones.

Para el propósito de este estudio se utilizó la definición de ideas principales de Aulls (1978):

la idea principal de un párrafo indica al lector la afirmación más importante que presenta el autor para explicar el tema (el tema se ha definido previamente como «aquello que indica al lector el tema del discursom). Este juicio o afirmación caracteriza a la idea principal, a la que se refieren la mayoría de las frases. La afirmación se formaliza generalmente en una frase. Una idea 
Lección 1: Ideas principales y detalles en párrafos-Explicitos. A los estudiantes se les enseña a identificar una idea explícita principal en un párrafo (frase-tema) y a asociar los detalles del párrafo que complementan la idea principal.

Lección 2: Ideas principales y detalles en pärrafos-Implicitos. Se les enseña a identificar una idea principal implícita de un párrafo y a asociar los detalles que complementan esa idea.

Lección 3: Revisión, refuerzo y prácticas de las lecciones 1 y 2. Se les da a los estudiantes un repaso de la instrucción dada para encontrar las ideas explicitas e implicitas principales en los párrafos y a asociar los detalles que complementan esas ideas.

Lección 4: Ideas principales y defalles en textos-Explicitos. A los estudiantes se les enseña a identificar una idea principal explícita (planteamiento del tema) en un texto corto. También se les enseña a confirmar estos planteamientos asociandolos con las ideas principales del texto (por ejemplo, las ideas principales de los párrafos se convierten en los "detalles" que mejor complementan al tema).

Lección 5: Ideas principales $y$ detalles en textos-Implicitos. A los estudiantes se les enseña a identificar una idea principal implícita (planteamiento del tema) de un texto corto. También se les enseña a confirmar estos planteamientos asociándolos a las ideas principales de los párrafos.

Lección 6: Resumen de las ideas principales de textos-Explicitas. A los estudiantes se les enseña a examinar los textos cortos y a hacer un resumen de las ideas principales, que consista en un planteamierto total del tema (explícito en el texto) y en los detalles que lo complementan (ideas principales explícitas del párrafo).

Lección 7: Resumen de las ideas principales de textos-lmplicitas. A los estudiantes se les enseña a examinar textos cortos y a realizar un resumen de la idea principal, que consista en un planteamiento total del tema (implicito en el texto) y en los detalles que lo complementan (una combinación de las ideas principales implicitas y explícitas de un párrafo).

Lección 8: Revisión, refuerzo y prácticas de todas las destrezas de ideas principales. Se enseña el repaso de la instrucción y práctica de todas las destrezas de ideas principales de las 7 lecciones anteriores.

principal implícita puede inferirse de la relación dominante entre los temas supraordenados y los subordinados de un párrafo (p. 92).

Se establecieron las siguientes definiciones operativas de diversos términos sobre ideas principales cuando se usaron para construir las ocho lecciones del grupo de Estrategia y escribir los postest:

Idea principal explícita de un párrafo: un párrafo que posee una frase-tema clara (lección 1).

Idea principal implicita de un párrafo: un párrafo que no contiene una frase-tema, pero sí una urelación dominante» que deducirse fácilmente de los «temas subordinados» (detalles) del párrafo (lección 2).

Idea principal explicita de un texto: un texto corto (de tres a cinco párrafos) que posee una frase-tema clara para todo el texto. Las ideas principales de los párrafos son subordinadas (actúan como «detalles») a la idea principal del texto (lección 4).

Idea principal implicita de un texto: un texto corto (de tres a cinco párrafos) que no contiene una frase-tema para todo el texto, pero sí una "relación dominante» que puede deducirse fácilmente de los «temas subordinados» (ideas principales de los párrafos) en el texto (lección 5).
Resumen de la idea principal del texto-explicita: un resumen simple de dos niveles en los que la idea principal explícita del texto, reside en el nivel supraordenado, y las ideas principales de los párrafos (explícitas en el texto) residen en el nivel subordinado (lección 6).

Resumen de la idea principal del texto-implicita: un resumen simple de dos niveles, en el que la idea principal implícita del texto, reside en un nivel supraordenado, y las ideas principales de los párrafos (bien explícitas, bien implícitas) residen en un nivel subordinado (lección 7).

Algunos ejemplos de textos que contienen esta variedad de ideas principales pueden encontrarse en el Apéndice B, que muestra los postests usados en este estudio.

Las listas de ideas principales de Dishner y Readence (1977), Jolly (1967) y Pearson y Johnson (1978) se utilizaron como guías para desarrollar la jerarquía de ocho lecciones usadas en este estudio, pero hay algunas características que distinguen esta lista de la secuencia de instrucción de ideas principales. En primer lugar, se enseña a los estudiantes a identificar los detalles complementarios junto con la instrucción para identificar las ideas principales, y no como entidades separadas como se hace habitualmente. 
En segundo lugar, se usan muchos heurísticos para ayudar a los estudiantes a adquirir las destrezas de las ideas principales (por ejemplo, la idea principal es un paraguas y los detalles complementarios lo que hay debajo; o bien, la idea principal es una mesa y los detalles complementarios sus patas). En tercer lugar, puesto que los trabajos realizados indican que el hecho de que los niños tengan que generar las respuestas trae como consecuencia una mejor comprensión que la obtenida cuando simplemente se conocen las respuestas correctas (Bridge, Belmore, Moskow, Cohen y Matthews, 1984; Doctrow, Witrock y Marks, 1978; Taylor y Berkowitz, 1980), se pidió a los alumnos que escribieran regularmente y hasta el final de la instrucción juicios o expresiones de las ideas principales. Y, por último, puesto que muchos lectores, especialmente los menos capaces, tienen problemas al resumir un texto, es decir, al transmitir los puntos principales de una forma concisa (Brown y Day, 1983; Tierney y Bridge, 1979; Winograd, 1984), se incluyó la instrucción en esta destreza en nuestra secuencia (lecciones 6 y 7).

La estrategia de instrucción en sí misma (Baumann, 1983b) estaba compuesta de cinco pasos que se repitieron en cada una de las ocho lecciones. A continuación se ofrece una breve descripción de cada paso. En el Apéndice A hay una transcripción completa de una de las lecciones de este estudio (lección 2).

1. Introducción: en este paso, a los estudiantes se les daba el objetivo de la lección y se les decia por qué la adquisición de esa destreza les ayudaría a ser mejores lectores. En realidad, la introducción era simplemente una panorámica estructurada de la lección, para que los estudiantes tuvieran una idea clara de su contenido y de su propósito.

2. Ejemplo: como una prolongación de la introducción, se mostraba una parte del texto que contuviera un ejemplo de la destreza a aprender. Esto ayudaba a que los estudiantes entendieran por completo lo que iban a aprender. El ejemplo demostraba cómo esta relación existe en un texto escrito (las ideas principales en este caso) y cómo la aptitud para entender la relación les ayudaría a comprender mejor lo que habian leido.
3. La instrucción Directa: la instrucción directa, el punto central de la estrategia de instrucción, consistía en que el profesor dijera, mostrara, demostrara y controlara la destreza que se iba a enseñar. En la instrucción directa el profesor se encargaba de la lección; la responsabilidad de la adquisición de la destreza descansaba únicamente en el profesor. Los estudiantes debian estar implicados en el aprendizaje, pero la transferencia de la responsabilidad a los alumnos se producía en los pasos siguientes. Los heurísticos también eran una parte importante de la instrucción directa; proporcionaban a los estudiantes una estructura o un método al que remitirse cuando en el futuro tuvieran que utilizar esa destreza.

4. Aplicación Dirigida por el Maestro: en este paso la responsabilidad de la adquisición de la destreza comienza a desplazarse al estudiante. En la aplicación dirigida por el maestro, el profesor todavía inicia las actividades, pero se pide al alumno que «descifre» los textos que contienen la destreza-objeto. En otras palabras, el profesor sigue estando presente para guiar y proporcionar un feedback, pero se fuerza a los estudiantes a aplicar la destreza enseñada anteriormente. Esto permite que el profesor tenga un control del éxito de los estudiantes en la adquisición de la destreza y que pueda volver a enseñarla si es necesario.

5. Práctica Independiente: en el último paso, se da la transición de la responsabilidad del maestro en el aprendizaje a una responsabilidad compartida por el profesor y el alumno y finalmente a una responsabilidad total del estudiante. Se ofrecen a los niños unos ejercicios que consisten en material no usado en la instrucción o en la aplicación y se les pide que practiquen esa destreza de forma independiente.

Grupo Básico. Para poder comparar la ejecución de los estudiantes del grupo de Estrategia con los niños que recibían una instrucción más tradicional sobre la comprensión de la idea principal, los estudiantes del grupo Básico recibieron una instrucción sobre las ideas principales y sobre los detalles complementarios tal y como venía indicado en el manual del profesor de una serie de libros básicos de lectura (Ride the Sunrise, nivel 12, 
del Ginn Reading Program, Clymer y Venezky, 1982). Se señalaron ocho lecciones distintas de la edición del profesor del Ride the Sunrise (pp. 30-31, 42-43, 54 , 64-65, 152-153, 280-281, 594-595, 625) del modo que indicaba el manual. Estos temas constituian la totalidad de las lecciones menos una, que sobre ideas principales y sobre detalles complementarios se dan en este nivel. Todas las páginas de ejercicios del Studybook y del Skillpack que lo acompañaban (libros de trabajo), se incluyeron como parte de la lección, según las recomendaciones del manual del profesor.

Grupo Control. Los estudiantes del grupo Control participaron en 8 sesiones sobre desarrollo de vocabulario significativo. El contenido de estas lecciones se adaptó de los ejercicios de Johnson y Pearson (1978, cap. 3). Los títulos de las lecciones especificas, en el orden en que fueron administradas, eran los siguientes: lista de atributos semántico/plano semántico, plano semántico-avanzado, análisis de las características semánticas-básico, análisis de las caracteristicas semánticasavanzado, categorización (palabras que van juntas), cómo se diferencian las palabras, analogías y palabras con varios significados.

\section{Medidas Dependientes}

Se usaron cinco instrumentos para determinar la aptitud de los estudiantes para comprender las ideas principales y los detalles complementarios. A continuación se ofrece una breve descripción de cada instrumento. El Apéndice B contiene unas reproducciones de cada uno de estos instrumentos con las respuestas correctas dadas a las pruebas.

Prueba de ideas principales de un párrafo. Para determinar si los estudiantes eran capaces de identificar las ideas principales de un párrafo, el experimentador creó un test de 20 preguntas de elección múltiple. Diez preguntas estaban formadas por párrafos en los que habia ideas principales explícitas (frases-tema) y las otras diez eran párrafos con ideas principales implícitas. En el Apéndice B se muestra la primera página de esta prueba, en la que se incluyó un ejemplo y la pregunta 1; ambos contenían ideas principales explícitas en el párrafo.

Prueba de detalles complementarios. Para eva- luar cómo asociaban los estudiantes los detalles con las ideas principales, el experimentador diseñó un test de 15 preguntas de elección múltiple. Cada pregunta era una idea principal y la tarea de los estudiantes era encontrar dos de los seis detalles dados que complementaran esa idea. Un ejercicio práctico y las preguntas 1-3 se ofrecen en el Apéndice $\mathrm{B}$.

Prueba de ideas principales de un texto. Para determinar si los estudiantes podian comprender las ideas principales de textos cortos (de unos tres a cinco párrafos), el experimentador creó un test de 6 preguntas de elección múltiple, de las que tres estaban formadas por párrafos cortos con ideas principales explícitas y las otras tres consistian en textos con ideas principales implícitas. Los pasajes fueron seleccionados imitando o adaptando textos similares de libros escolares de ciencias sociales del grado 6. La página 1 de esta prueba se muestra en el apéndice B. El item 1 mide la idea principal explícita del texto; el item 2 mide la idea principal implícita.

Prueba del resumen de la idea principal. Esta prueba determinó si los estudiantes podían resumir un texto corto construyendo un esquema de las ideas principales del texto y del párrafo. Se pidió a los estudiantes que escribieran un planteamiento de la idea principal del texto y de las ideas principales de los párrafos subordinados correspondientes a esa idea principal del pasaje. Se usaron dos textos cortos (adaptados de libros de texto de ciencias sociales del grado 6). Los dos están en el apéndice $\mathrm{B}$. El primer texto contiene una idea principal explicita en el texto y unas ideas principales explícitas en los párrafos subordinados; el segundo pasaje contiene una idea principal implicita en el texto y unas ideas explícitas en los párrafos subordinados.

El experimentador y un ayudante puntuaron independientemente cada resumen. Se otorgó una puntuación completa a las ideas principales del texto o del párrafo que eran reproducciones exactas del texto (sólo para las ideas principales explícitas) o paráfrasis razonables. Se dio una puntuación parcial (medio punto) a las respuestas incompletas. Por ejemplo, se dio una puntuación completa a la idea principal del texto del item 1 para la respuesta «Por qué tenemos problemas alimentarios en el mundo" puesto que era una paráfrasis razonable; sin embargo, sólo se dio medio punto a la respuesta "problemas alimentarios en el mundo" porque proporcionaba sólo información parcial. Para la idea princi- 
pal implícita del texto, se construyeron a priori algunas respuestas correctas y se aplicó el mismo criterio de evaluación completa o parcial en la puntuación de estas preguntas. Se obtuvo un índice del acuerdo interjueces del 91,5 por 100 . La mayoria de las discrepancias en la puntuación se dieron en la puntuación parcial y todas las diferencias se salvaron en una reunión posterior.

Prueba de recuerdo libre. La última medida dependiente pedia a los estudiantes que leyeran. en silencio un texto breve (construido según una selección de textos de un libro de ciencias sociales del grado 6) y después de una tarea interviniente (laberintos y búsqueda de palabras) para controlar la memoria a corto plazo, que escribieran un recuerdo libre. El texto puesto a prueba estaba dividido en 30 unidades de ideas de acuerdo con el procedimiento de Thorndyke (1977), que esencialmente divide un texto en frases dependientes $e$ independientes. Las unidades de ideas resultantes se identifican por medio de fragmentos del texto de la prueba de recuerdo libre reproducido en el apéndice $B$, aunque la versión del texto que los estudiantes leyeron no estaba segmentada de esta forma. En dos ejemplos, las unidades de ideas que no se identificaron de acuerdo con el procedimiento de Thorndyke («o cartógrafos» en la línea 1 y alluvia y nieve» en la tercera línea empezando por el final) se clasificaron como unidades de ideas separadas, ya que estaban en aposición o en planteamientos entre paréntesis dentro de ideas separadas. Por lo tanto, estas dos ideas, designadas entre paréntesis, contaron como ideas separadas, incluso aunque residieran en una unidad de idea mayor. El pasaje de prueba de recuerdo libre contenía una idea principal explícita del texto y tres ideas principales explícitas del párrafo subordinado. El primero está marcado por un doble subrayado y el último con un subrayado sencillo en el apéndice $B$.

Las puntuaciones de los protocolos de recuerdo libre se obtuvieron comparando cada planteamiento del protocolo escrito con las unidades de ideas del texto segmentado y adjudicando una puntuación total, parcial o nula. Se obtuvieron dos puntuaciones en cada protocolo: recuerdo total de las ideas principales (ideas principales del texto más ideas principales del párrafo) y recuerdo total de las unidades de ideas. Para obtener estas dos puntuaciones se utilizó el mismo criterio que el usado para evaluar los planteamientos de la idea principal del texto cuando se halló la puntuación en la prueba del resumen de la idea principal. El experimentador y el ayudante puntuaron independientemente los 66 protocolos y el acuerdo interjueces fue del 90,8 por 100 . Al igual que con la puntuación de las pruebas de resúmenes de las ideas principales, la mayoría de los desacuerdos provinieron de la adjudicación de las puntuaciones parciales y estas discrepancias se resolvieron en una reunión posterior.

\section{Análisis de Datos}

Puesto que se aplicaron diversas medidas dependientes para determinar la comprensión de la idea principal, se empleó un análisis de varianza multivariado (MANOVA) para medir los efectos del tratamiento y de los niveles de rendimiento. En el análisis MANOVA se introdujeron 9 puntuaciones separadas de los postests: (a) ideas principales explícitas del párrafo correctas en la prueba de Ideas Principales de un párrafo (puntuación máxima posible $=10$ ); (b) ideas principales implicitas del párrafo correctas, en la prueba de Ideas Principales de un párrafo (puntuación máxima $=10$ ); (c) detalles correctos en la prueba de Detalles complementarios (puntuación máxima $=30$ ); (d) ideas principales explicitas del texto correctas, en la prueba de Ideas Principales de un texto (puntuación máxima $=3$ ); (e) ideas principales implícitas del texto correctas, en la prueba de Ideas Principales de un texto (puntuación máxima $=3$ ); (f). ideas principales del párrafo correctas, en la prueba del Resumen de la Idea Principal (puntuación máxima $=6$ ); $(\mathrm{g})$ ideas principales del texto correctas, en la prueba del Resumen de Ideas principales (puntuación máxima $=2)$; $(\mathrm{h})$ ideas principales del texto y del párrafo correctas, en la prueba de Recuerdo Libre (puntuación máxima posible = 4); (i) ideas totales recordadas, en la prueba de Recuerdo Libre (puntuación máxima = 30).

Las cinco primeras puntuaciones se consideraron medidas directas de la comprensión del texto y del párrafo y por eso, nos referiremos a ellas como medidas de transmisión "cercanas". Las otras cuatro puntuaciones se consideraron como medidas más indirectas de la comprensión y las llamaremos medidas de transmisión «lejanas". Se realizaron dos MANOVA 3 (tratamiento: Estrategia, Básico y Control) por 3 (Nivel de rendimiento: Alto, Medio y Bajo), uno de ellos sobre las medidas de transmisión cercanas y otro sobre las medidas lejanas. Se realizaron pruebas univa- 
riadas sobre las medidas dependientes individuales de los MANOVA totales significativos.

\section{RESULTADOS}

\section{Medidas de transmisión cercanas}

La tabla 2 presenta las puntuaciones medias y las desviaciones típicas de la ejecución de los estudiantes (por nivel de rendimiento) en las medidas de transmisión cercanas. Los resultados del MAROVA ${ }^{2}$ revelaron un efecto significativo para el tratamiento, $F(10,106)$ $=6,797, \mathrm{p}<0,001$ y para el nivel de rendimiento, $F(10,106)=5,283, p<0,001$. La interacción nivel de rendimiento $\mathrm{X}$ tratamiento no alcanzó significación estadistica, $\mathrm{F}(20,177)=0,658, \mathrm{p}<0,863$.

La tabla 3 muestra los resultados de la prueba $F$ univariada y los resultados post hoc para el factor tratamiento en las medidas de transmisión cercanas. Como podemos ver, cada prueba univariada individual alcanzó significación estadística, lo que indica que cada variable dependiente contribuye al MANOVA total para el factor tratamiento. Las medidas post hoc indicaron que el grupo de Estrategia rindió mejor que el grupo Básico y que el de Control en cada variable dependiente; además, la ejecución del grupo básico sobrepasó la del grupo Control en el subtest de ideas principales explícitas de un párrafo.

TABLA 2

Puntuaciones medias ( $y$ desviaciones típicas) para las medidas de transmisión cercanas

\begin{tabular}{|c|c|c|c|c|c|c|c|c|c|c|c|c|}
\hline & \multicolumn{4}{|c|}{ Estrategia } & \multicolumn{4}{|c|}{ Básico } & \multicolumn{4}{|c|}{ Control } \\
\hline & bajo & medio & alto & total & bajo & medio & alto & total & bajo & medio & alto & total \\
\hline $\begin{array}{l}\text { Ideas principale } \\
\text { explícitas } \\
\text { del párrafo } \\
\text { Ideas orincinales }\end{array}$ & $\begin{array}{r}7,00 \\
(1,83)\end{array}$ & $\begin{array}{r}8,13 \\
(1,36)\end{array}$ & $\begin{array}{r}8,86 \\
(0,90)\end{array}$ & $\begin{array}{r}8,80 \\
(1,54)\end{array}$ & $\begin{array}{r}4,71 \\
(1,38)\end{array}$ & $\begin{array}{r}6,25 \\
(1,67)\end{array}$ & $\begin{array}{r}7,57 \\
(1,51)\end{array}$ & $\begin{array}{r}6,18 \\
(1,74)\end{array}$ & $\begin{array}{r}4,71 \\
(1,80)\end{array}$ & $\begin{array}{r}4,63 \\
(1,19)\end{array}$ & $\begin{array}{r}6,57 \\
(1,62)\end{array}$ & $\begin{array}{r}5,27 \\
(1,72)\end{array}$ \\
\hline $\begin{array}{l}\text { implicitas } \\
\text { del párrafo } \\
\text { Detalles } \\
\text { Complemen. } \\
\text { Ideas principales }\end{array}$ & $\begin{array}{r}5,57 \\
(1,13) \\
23,57 \\
(3,87)\end{array}$ & $\begin{array}{c}6,50 \\
(1,07) \\
26,88 \\
(3)\end{array}$ & $\begin{array}{r}7,71 \\
(2,06) \\
28,57 \\
(1,27)\end{array}$ & $\begin{array}{r}6,59 \\
(1,65) \\
26,37 \\
(3,47)\end{array}$ & $\begin{array}{r}3,43 \\
(1,72) \\
19,43 \\
(4,67)\end{array}$ & $\begin{array}{r}5,25 \\
(1,75) \\
23,25 \\
(2,61)\end{array}$ & $\begin{array}{r}6,43 \\
(1,72) \\
25,43 \\
(1,40)\end{array}$ & $\begin{array}{r}5,05 \\
(2,06) \\
22,73 \\
(3,83)\end{array}$ & $\begin{array}{r}3,14 \\
(1,68) \\
19,14 \\
(3,24)\end{array}$ & $\begin{array}{r}4,50 \\
(2,20) \\
21,75 \\
(3,15)\end{array}$ & $\begin{array}{r}5,14 \\
(1,95) \\
24,43 \\
(1,27)\end{array}$ & $\begin{array}{r}4,27 \\
(2,05) \\
21,77 \\
(3,38)\end{array}$ \\
\hline $\begin{array}{l}\text { explícitas } \\
\text { del texto }\end{array}$ & $\begin{array}{r}2,57 \\
(0,79)\end{array}$ & $\begin{array}{l}3 \\
0\end{array}$ & $\begin{array}{l}3 \\
0\end{array}$ & $\begin{array}{l}2,86 \\
0,47\end{array}$ & $\begin{array}{l}1,57 \\
0,98\end{array}$ & $\begin{array}{l}2,50 \\
0,54\end{array}$ & $\begin{array}{l}2,14 \\
0,38\end{array}$ & $\begin{array}{l}2,09 \\
0,75\end{array}$ & $\begin{array}{l}1,57 \\
0,98\end{array}$ & $\begin{array}{l}1,75 \\
0,71\end{array}$ & $\begin{array}{l}2,29 \\
0,76\end{array}$ & $\begin{array}{l}1,86 \\
0,83\end{array}$ \\
\hline $\begin{array}{l}\text { implícitas } \\
\text { del texto }\end{array}$ & $\begin{array}{r}2,43 \\
(0,54)\end{array}$ & $\begin{array}{r}2,63 \\
(0,74)\end{array}$ & $\begin{array}{r}2,57 \\
(0,79)\end{array}$ & $\begin{array}{r}2,55 \\
(0,67)\end{array}$ & $\begin{array}{r}1,86 \\
(0,90)\end{array}$ & $\begin{array}{r}1,63 \\
(0,52)\end{array}$ & $\begin{array}{r}2,57 \\
(0,79)\end{array}$ & $\begin{array}{r}2,00 \\
(0,82)\end{array}$ & $\begin{array}{r}1,14 \\
(0,90)\end{array}$ & $\begin{array}{r}1,63 \\
(1,06)\end{array}$ & $\begin{array}{r}2,14 \\
(0,38)\end{array}$ & $\begin{array}{r}1,64 \\
(0,90)\end{array}$ \\
\hline
\end{tabular}

PPuntuación máxima $=10$

${ }^{\text {bPuntuación máxima }}=30$

${ }^{\mathrm{C}}$ Puntuación máxima $=3$

TABLA 3

Pruebas F Univariadas y procedimientos post boc para el factor tratamiento en las medidas de transmision cercanas

\begin{tabular}{|c|c|c|c|c|c|}
\hline \multirow[b]{2}{*}{ Variables } & \multicolumn{3}{|c|}{ Prueba F Univariada } & \multicolumn{2}{|c|}{ Post Hoc } \\
\hline & $\mathbf{F}$ & df & $\mathbf{p}$ & contraste $^{b}$ & $\mathbf{p}$ \\
\hline Ideas Principales Explícitas del párrafo & 20,599 & 2,57 & 0,001 & $\begin{array}{l}S>B \\
S>C \\
B>C\end{array}$ & $\begin{array}{l}0,01 \\
0,01 \\
0,05\end{array}$ \\
\hline Ideas Principales Implicitas del párrafo & 10,170 & 2,57 & 0,001 & $\begin{array}{l}S>B \\
S>C\end{array}$ & $\begin{array}{l}0,01 \\
0,01\end{array}$ \\
\hline Detalles Complementarios & 15,227 & 2,57 & 0,001 & $\begin{array}{l}\mathrm{S}>\mathrm{B} \\
\mathrm{S}>\mathrm{C}\end{array}$ & $\begin{array}{l}0,01 \\
0,01\end{array}$ \\
\hline Ideas Principales Explicitas del texto & 13,823 & 2,57 & 0,001 & $\begin{array}{l}S>B \\
S>C\end{array}$ & $\begin{array}{l}0,01 \\
0.01\end{array}$ \\
\hline Ideas Principales Implícitas del texto & 7,875 & 2,57 & 0,001 & $\begin{array}{l}S>B \\
S>C\end{array}$ & $\begin{array}{l}0,01 \\
0,01\end{array}$ \\
\hline
\end{tabular}

en los procedimientos post hoc se utilizó la prueba de Newman-Keuls.

b $\mathrm{S}=$ Estrategia; $\mathrm{B}=$ básico; $\mathrm{C}=$ control. 
Aunque el propósito primordial de este estudio era evaluar el efecto del método de instrucción de ideas principales (tratamiento), hay que señalar que el MANOVA total significativo para los niveles de rendimiento estaba apoyado en las pruebas $F$ univariadas significativas ( $p \leqslant 0,029$ ) para cada una de las variables dependientes individuales, lo que indica un efecto importante del nivel de rendimiento para cada medida de transmisión cercana.

\section{Medidas de transmisión lejanas}

La tabla 4 muestra las puntuaciones medias y las desviaciones típicas en la ejecución de los sujetos (por nivel de rendimiento) en las medidas de transmisión lejanas. Los resultados del MAROVA revelaron un efecto principal significativo para el tratamiento, $\mathrm{F}(8,108)=2,919, \mathrm{p}<0,005$ y para el nivel de rendimiento, $\mathrm{F}(8,108)=4,013, \mathrm{p}<$ 0,001 . La interacción nivel de rendimiento $X$ tratamiento no alcanzó significación estadística, $\mathrm{F}(16,166)=1,085, \mathrm{p}<0,373$.

La tabla 5 muestra los resultados de las pruebas $F$ univariadas y los resultados post hoc para el factor tratamiento en las medidas de transmisión lejanas. Como puede observarse, las dós medidas de las pruebas del tesumen fueron las únicas medidas de transmisión lejanas que contribuyeron al MANOVA total significativo para el tratamiento, puesto que ninguna de las medidas de recuerdo libre alcanzó significación estadística.

Las pruebas post hoc para el resumen de las ideas principales de un párrafo y de un texto revelaron que el grupo de Estrategia rindió mejor que los grupos Básico y Control en cada una de estas medidas. EI MAROVA total significativo para el nivel de rendimiento estaba apoyado en las pruebas $F$ univariadas significativas $(p \leqslant 0,01)$ en cada una de las cuatro variables, lo que de nuevo indica un gran efecto del nivel de rendimiento en cada medida de transmisión lejana.

TABLA 4

Puntuaciones medias ( $y$ desviaciones tipicas) para las medidas de transmisión lejanas

\begin{tabular}{|c|c|c|c|c|c|c|c|c|c|c|c|c|}
\hline & \multicolumn{4}{|c|}{ Estrategia } & \multicolumn{4}{|c|}{ Básico } & \multicolumn{4}{|c|}{ Control } \\
\hline & jo & medio & alto & tal & jo & lio' & Ito & total & ajo & redio & alto & total \\
\hline \multirow{5}{*}{$\begin{array}{l}\text { Ideas principales } \\
\text { del párrafo } \\
\text { (resumen) }^{\mathrm{a}} \\
\text { Ideas principales }_{\text {del texto }} \\
\text { (resumen) }^{\mathrm{b}} \\
\text { Ideas principales }_{\text {en el recuerdo }} \\
\text { libre }^{\mathrm{c}} \\
\text { Ideas totales } \\
\text { en el recuerdo } \\
\text { libred }\end{array}$} & & & & & & & & & & & & \\
\hline & $\begin{array}{c}4,14 \\
(0,48)\end{array}$ & $\begin{array}{c}4,31 \\
(1,75)\end{array}$ & $\begin{array}{c}4,86 \\
(0,95)\end{array}$ & $\begin{array}{c}4,44 \\
(1,20)\end{array}$ & $\begin{array}{c}2,07 \\
(1,92)\end{array}$ & $\begin{array}{c}3,31 \\
(1,39)\end{array}$ & $\begin{array}{c}3,43 \\
(2,07)\end{array}$ & $\begin{array}{c}2,96 \\
(1,82)\end{array}$ & $\begin{array}{c}1,79 \\
(1,35)\end{array}$ & $\begin{array}{c}1,63 \\
(1,62)\end{array}$ & $\begin{array}{c}4,29 \\
(1,35)\end{array}$ & $\begin{array}{c}2,52 \\
(1,86)\end{array}$ \\
\hline & $\begin{array}{c}1,21 \\
(0,49)\end{array}$ & $\begin{array}{c}1,44 \\
(0,73)\end{array}$ & $\begin{array}{c}1,79 \\
(0,39)\end{array}$ & $\begin{array}{c}1,48 \\
(0,59)\end{array}$ & $\begin{array}{c}0,71 \\
(0,39)\end{array}$ & $\begin{array}{c}1,19 \\
(0,65)\end{array}$ & $\begin{array}{c}1,14 \\
(0,63)\end{array}$ & $\begin{array}{c}1,02 \\
(0,59)\end{array}$ & $\begin{array}{c}0,64 \\
(0,48)\end{array}$ & $\begin{array}{c}0,75 \\
(0,46)\end{array}$ & $\begin{array}{c}1,29 \\
(0,76)\end{array}$ & $\begin{array}{c}0,89 \\
(0,62)\end{array}$ \\
\hline & $\begin{array}{c}1,43 \\
(0,89)\end{array}$ & $\begin{array}{c}2,06 \\
(0,12)\end{array}$ & $\begin{array}{c}2,07 \\
(0,93)\end{array}$ & $\begin{array}{c}1,86 \\
(0,10)\end{array}$ & $\begin{array}{c}0,79 \\
(0,57)\end{array}$ & $\begin{array}{c}1,19 \\
(1,00)\end{array}$ & $\begin{array}{c}2,14 \\
(0,99)\end{array}$ & $\begin{array}{c}1,36 \\
(0,10)\end{array}$ & $\begin{array}{c}0,71 \\
(0,64)\end{array}$ & $\begin{array}{c}0,69 \\
(0,65)\end{array}$ & $\begin{array}{c}2,57 \\
(0,12)\end{array}$ & $\begin{array}{c}1,30 \\
(0,12)\end{array}$ \\
\hline & $\begin{array}{c}3,79 \\
(3,49)\end{array}$ & $\begin{array}{c}6,94 \\
(2,98)\end{array}$ & $\begin{array}{c}6,79 \\
(3,45)\end{array}$ & $\begin{array}{c}5,89 \\
(3,47)\end{array}$ & $\begin{array}{c}2,36 \\
(2,50)\end{array}$ & $\begin{array}{c}3,63 \\
(2,15)\end{array}$ & $\begin{array}{c}7,43 \\
(2,57)\end{array}$ & $\begin{array}{c}4,43 \\
(3,15)\end{array}$ & $\begin{array}{c}3,79 \\
(1,50)\end{array}$ & $\begin{array}{c}3,75 \\
(1,56)\end{array}$ & $\begin{array}{c}6,64 \\
(2,81)\end{array}$ & $\begin{array}{c}4,68 \\
(2,36)\end{array}$ \\
\hline
\end{tabular}

${ }^{2}$ Puntuación máxima $=6$

buntuación máxima $=2$

'Puntuación máxima $=4$

dPuntuación máxima $=30$

TABLA 5

Pruebas F Univariadas y procedimientos post hoc para el factor tratamiento en las medidas de transmisión lejanas

\begin{tabular}{|c|c|c|c|c|c|}
\hline \multirow[b]{2}{*}{ Variables } & \multicolumn{3}{|c|}{ Prueba F Univariada } & \multicolumn{2}{|c|}{ Post Hoc ${ }^{2}$} \\
\hline & $\mathbf{F}$ & df & $\mathbf{p}$ & contraste $^{b}$ & $\mathbf{p}$ \\
\hline Ideas Principales del párrafo en el resumen & 6,448 & 2,57 & 0,003 & $\begin{array}{l}S>B \\
S>C\end{array}$ & $\begin{array}{l}0,01 \\
0,01\end{array}$ \\
\hline Ideas Principales del texto en el resumen & 9,682 & 2,57 & 0,001 & $\mathrm{~S}>\mathrm{B}$ & 0,01 \\
\hline $\begin{array}{l}\text { Ideas principales en el recuerdo libre } \\
\text { Ideas totales en el recuerdo libre }\end{array}$ & $\begin{array}{l}2,462 \\
1,926\end{array}$ & $\begin{array}{l}2,57 \\
2,57\end{array}$ & $\begin{array}{l}0,094 \\
0,155\end{array}$ & $\mathrm{~S}>\mathrm{C}$ & 0,01 \\
\hline
\end{tabular}

a los procedimientos post hoc se utilizó la prueba de Newman-Keuls.

b $\mathrm{S}=$ Estrategia; $\mathrm{B}=$ básico; $\mathrm{C}=$ control. 


\section{DISCUSION}

Los resultados de este estudio sugieren firmemente que la aplicación de un modelo de instrucción directa para enseñar las destrezas de la comprensión lectora de las ideas principales es superior a la aplicación de la instrucción por medio de libros de lectura y a la de actividades de desarrollo de vocabulario significativó. Podemos resumir los resultados de! siguiente modo:

1. El grupo de Estrategia rindió mejor que el grupo Básico y que el de Control en la aptitud para reconocer las ideas principales explícitas o implicitas de un párrafo, para reconocer los detalles complementarios, para reconocer las ideas principales explícitas e implícitas de un texto y para resumir las ideas principales de un párrafo y de un texto.

2. El grupo Básico rindió mejor que el grupo Control en la aptitud para recoger las ideas principales explícitas de un párrafo.

3. El tratamiento de los grupos no afectó a la aptitud para las ideas principales en el recuerdo ni en el recuerdo libre total.

4. Se observó un fuerte efecto del nivel de rendimiento, lo que indica que los lectores más capaces rinden mejor que los lectores menos capaces, en todas las variables dependientes.

5. No hubo interacciones entre el tratamiento y el nivel de rendimiento.

La ejecución superior del grupo de Estrategia, comparada con la del grupo Control, fue satisfactoria pero no sorprendente, puesto que el sentido común nos dice que los estudiantes que han recibido una instrucción directa o intensiva sobre cómo comprender las ideas principales, han de ejecutar mejor la tarea que sus compañeros que no han recibido una instrucción similar. Sin embargo, los efectos del tratamiento a favor del grupo de Estrategia indican que las destrezas sobre las ideas principales pueden enseñarse eficazmente cuando la instrucción es directa y sistemática, lo cual está de acuerdo con los éxitos anteriores de los estudios de comprensión de método directo (Hansen, 1981; Hansen y Pearson, 1983; Patching et al., 1983; ver las revisiones de Pearson, 1982; 1984b; Pearson y Gallagher, 1983; Raphael y Pearson, 1982; Tharp, 1982).

Fue sin embargo, sorprendente la aptitud del grupo de Estrategia para rendir mejor que el grupo Básico en todas las medias de transmisión cercana y en las dos puntuaciones de la prueba del Resumen de Ideas principales en el caso de las medidas de transmisión lejana, especialmente en algunas variables dependientes. Podría esperarse que el grupo de Estrategia tuviera más destreza en reconocer las ideas principales explícitas de un texto y en completar un resumen de ideas principales puesto que la instrucción sobre cómo intensificar las ideas principales de un texto y sobre cómo hacer un resumen, no se incluía en el libro básico de lectura usado (al contrario que el grupo de Estrategia que recibió dos lecciones de cada estrategia). La ejecución superior del grupo de Estrategia al reconocer las ideas principales explícitas e implícitas de un párrafo, fue inesperada. El libro básico de lectura usado en este estudio, tenía muchas lecciones sobre cómo identificar las ideas principales de un párrafo y los detalles complementarios; de hecho, todas las lecciones hásicas de algún modo se centraban en estas destrezas. Por lo tanto, el contenido de las lecciones no explica por 'sí solo, la ejecución superior del grupo de Estrategia.

Una explicación obvia es que puesto que el modelo de instrucción directa ofrece mayor cantidad de uinstrucción directa» que la serie de los libros de lectura (Durkin, 1981), los estudiantes del grupo de Estrategia adquirieron estas destrezas con mayor profundidad y comprensión. Aunque las críticas de Durkin (1981) a los libros básicos de lectura estaban justificadas respecto a los manuales de 1978 y 1979 que ella examinó, hay que señalar que el programa usado en este trabajo, así como otras series de ediciones más-modernas, ofrecen a los profesores un componente de enseñanza relativamente eficaz. En otras palabras, el programa aplicado al grupo Básico no estaba desprovisto de instrucción directa en la comprensión de ideas principales. Lo que diferenciaba el modelo de instrucción directa de las lecciones básicas usadas en esta investigación, es el énfasis 
puesto en la aplicación y en la práctica en nuevos contextos, más que en la presencia de estrategias de "enseñanza" más eficaces. Ya que a los estudiantes del grupo de Estrategia se les dieron muchas oportunidades de practicar lo que se les había enseñado, podían transferir (Baumann, 1983d; Brown, Campione y Day, 1981) la destreza para la idea principal aprendida y para los detalles, a un nuevo contexto, es decir, al postest. Lo que también distinguía el modelo de instrucción directa de la del grupo Básico era el énfasis dado al hecho de que los estudiantes generaran respuestas (escribir el planteamiento de las ideas principales) opuesto al hecho de que simplemente reconocieran las respuestas correctas (subrayarlas o rodearlas); esa primera alternativa es una técnica de instrucción más eficaz (Doctrow, Wittrock y Marks, 1978; Taylor y Berkowitz, 1980). De hecho, un trabajo reciente de Bridge et al. (1984) reveló que la composición de una frase-idea principal después de leer una selección de texto cortos, tuvo como resultado una mayor memoria en lectores adultos buenos y deficientes, cuando se les comparó con sujetos que solamente indicaron la comprensibilidad de los textos que habia leido.

La ausencia de estas características en las lecciones, puede ayudarnos a comprender la ejecución relativamente pobre del grupo Básico en comparación con la del grupo Control (el grupo Básico sólo rindió mejor en la aptitud para reconocer las ideas principales explícitas de un párrafo). En otras palabras, la falta de práctica y de tareas de transferencia, así como de las actividades que generen respuestas, dio la transición de la responsabilidad total del profesor en el aprendizaje a la del estudiante en la adquisición de la destreza (Baumann, 1983b; Pearson y Callagher, 1983). Una hipótesis acerca de la competencia de los maestros que explique la pobre ejecución del grupo Básico -es decir, que los profesores no sigan regularmente la instrucción tal y como la señalan los manuales (Durkin, 1983; Mason, 1983) - no es aplicable en este ejemplo, puesto que el experimentador siguió fielmente todas las indicaciones del manual básico para la instrucción de la destreza. La comparación entre el rendimiento de los estudiantes cuyos de otros alumnos cuyos maestros se desvían de las instrucciones prescritas, podría ser una línea de investigación futura necesaria para determinar si hay una relación entre estas conductas y la adquisición de la aptitud para comprender.

Una explicación posible del fracaso para descubrir las diferencias entre los grupos en la prueba de recuerdos libres, podria ser la falta de familiaridad de los estudiantes en esta tarea. Ninguno de los tratamientos incluyó ejercicios de recuerdo libre, por lo tanto, no fue sorprendente que la cantidad de recuerdo no difiriera según el tratamiento aplicado. Este abandono de la tarea de recuerdo libre puede explicar también la poca cantidad de información contenida en los protocolos de recuerdo (las ideas totales recordadas fueron, 20 por 100,15 por 100 y 16 por 100 para los grupos de Estrategia, Básico y Control, respectivamente).

La prueba de recuerdo libre se incluia en una batería de medidas dependientes para determinar si los estudiantes del grupo de Estrategia podian ser más sensibles a las ideas principales de un párrafo o de un texto que los sujetos de los grupos Básico o Control. Desde luego, la ejecución del grupo de Estrategia en las medidas dependientes sugería que estos estudiantes tenían más destreza en la comprensión de la idea principal; esta aptitud no se manifestaba solamente en el ejercicio de recuerdo libre. Es interesante señalar que, a pesar de que las instrucciones dadas para realizar la prueba de recuerdo libre indicaban que había de hacerla en forma de párrafo, varios de los recuerdos libres de alumnos de grupo de Estrategia estaban en forma de resumen, aparentemente como un reflejo de las instrucciones anteriores dadas en la prueba de resumen. Tal vez, si se hubiera pedido incluir en la prueba de recuerdo de tantas ideas "principales» del texto como fuera posible, la ejecución del grupo de Estrategia en esta tarea hubiera sido mejor.

\section{CONCLUSIONES}

Hay que señalar algunas limitaciones de este sentido. En primer lugar, aunque se intentó crear un ambiente de instruc- 
ción tan natural o ecológicamente válido (Bronfenbrener, 1976) como fuera posible, toda la instrucción la administró una persona desconocida y ajena a los estudiantes. Las investigaciones posteriores necesitarian determinar si se puede entrenar a los profesores a adquirir la destreza necesaria para aplicar un modelo de instrucción directa. El éxito que Hansen y Pearson (1983) tuvieron al enseñar a maestros a adquirir estrategias para enseñar la comprensión, es un buen modelo para continuar la investigación sobre este tema.

Una segunda limitación es que las lecciones dadas al grupo Básico se presentaron en un orden temporal distinto del que pretendía según los autores de los libros de lectura, es decir, las lecciones, se presentaron de una forma densa y no durante un período largo de tiempo como indicaba el manual. Aunque puede argüirse que hacer esto podría haber aumentado el nivel de ejecución de los miembros del grupo Básico en las medidas dependientes, no es la forma habitual de usar los libros de lectura. Para determinar si la aplicación del modelo de instrucción directa es eficaz a largo plazo, debería realizarse un estudio de un año de duración en una clase en donde un grupo de estudiantes que recibe instrucción básica de la comprensión tal y como está prescrito en el manual del profesor se compara con un segundo grupo al que se le administra instrucción básica y en donde las lecciones deficientes (con respecto al modelo de instrucción directa) fueran ampliada o mejorada (Baumann, 1983c, 1983d).

Resumiendo, los resultados de este trabajo son coherentes con las investigaciones generalizadas sobre el efecto positivo de la «instrucción directa" (Berliner, 1981; Brophy, 1979; Duffy, 1981, 1983; Fisher et al., 1980) y con los resultados de los estudios anteriores sobre los éxitos de la instrucción de la comprensión (Hansen, 1981; Hansen y Pearson, 1983; Patching et al., 1983; Raphael y Pearson, 1982; Tharp, 1982). Seria necesario para determinar a gran escala si la aplicación de los principios de instrucción directa (como los implantados en este estudio) son eficaces a la hora de mejorar la comprensión de los estudiantes de las ideas principales y de otras aptitudes para la comprensión, llevar a cabo algunos estudios - similares al Project Follow Through (Becker, 1977) - que amplien esta línea de investigación en otros contextos educativos más naturales.

\section{Referencias}

Alexandi:R, C. F.: «Strategies for finding the main idea». Journal of Reading, 1976, 19, 299-301.

Armbruster, B. B., Stevens, R. J., y Rosenshine, B.: Analyzing content coverage and emphasis: $A$ study of three curricula and two tests (Technical Report No. 26). Champaign, IL: Universidad de Illinois, Center for the Study of Reading, 1977.

Aulls, M. W.: Developmental and remedial reading in the middle grades. Boston: Allyn \& Bacon, 1978.

AXELROD, J.: "Getting the main idea is still the main idea". Journal of Reading, 1975, 18, 383-387.

BAkER, L., y STEIN, N.: "The development of prose comprehension skills". En C. M. Santa \& B. L. Hayes (Eds.), Children's prose comprehension: Researcb and practice (Pp. 7-13). Newark, DE: International Reading Association, 1981.

BAUMANN, J. F.: "Effect of ideational prominence on children's reading comprehension of expository prosem. Journal of Reading Bebavior, 1981, 13, 49-56.

BAUMANN, ]. F.: "Research on children's main idea comprehension: A problem of ecological validity». Reading Psychology, 1982, 3, 167-177, 1982.

BaumanN, J. F.: «Children's ability to comprehend main ideas in content textbooks». Reading World, $1983 \mathrm{a}, 22,322-331$.

BAUMANN, J. F.: «A generic comprehension instructional strategy». Reading World, 1983b, 22, $284-294$.

Baumann, J. F.: How to improve comprebension instruction in basal readers. Paper presented at the Twentyeighth annual meeting of the International Reading Association. Anaheim, CA, 1983c.

BAUMANN, J. F.: «Six principles for the development of reading comprehension instructional methods and materials". Reading Improvement, 1983d, 20, 187-192.

BeCKER. W. C.: "Teaching reading and language to the disadvantaged-What we have learned from field research». Harvard Educational Review, 1977, 47, 518-543.

Berliner, D. C.: "Academic learning time and reading achievement». En J. T. Guthrie (Ed.), Comprebension and teacbing: Research Reviews (pp. 203-226). Nark, DE: International Reading Association, 1981.

Berliner, D. C., y Rosenshine, B. V.: "The acquisition of knowledge in the classroom». En R. C. Anderson, R. J. Spiro, y W. E. Montague (Eds.). Schooling and the acquisition of knowledge (pp. 375-396). Hillsdale, N. J.: Lawrence Erlbaum Associates, 1977.

Bridges, C. A.; Belmore, S. M.; Moskow, S. P., Cohen, S. S., y MatThews, P. D.: "Topicalization and memory for main ideas in prosen. Journal of Reading Bebavior, 1984, 16, 61-80. 
BRONFENBRENNER, U.: "The experimental ecology of education. Educational Rsearcher, 1976, 5(9), 5-15.

BROPHY, J.: "Teacher behavior and student learning". Educational Leadership, 1979, 37, 33-38.

BROPHY, J., y GOOD, T. L.: "Teacher behavior and student achievement». En M. C. Wittrock (Ed.), Third bandbook of research on teaching. Nueva York: Macmillan (en prensa).

Brown, A. L.; CAMpione, J. C., y DAY, J. D.: «Learning to learn: On training students to learn from texts». Educational Researcher, 1981, 10, 14-24.

BRown, A. L., y DAY, J. D.: Macrorules for summarizing texts: The development of expertise (Technical Report No. 270). Champaign, IL: Universidad de Illinois, Center for the Study of Reading, 1983.

Brown, A. L., y SMILFY, S. S.: «Rating the importance of structural units of prose passages: A problem of metacognitive development». Child Development, 1977, 48, 1-9.

Christie, D. J., y Schumacher, G. M.: «Development trends in the abstraction and recall of relevant versus irrelevant thematic information from connected verbal materials». Child Development, 1975, 46, 589-602.

Clymer, T., y Venezky, R. L.: Ginn Reading Program. Lexington, MA: Ginn and Company, 1982.

DANNER, F. W.: "Children's understanding of intersentence organization in the recall of short descriptive passages». Journal of Educational Psychology, 1976, 68, 174-183.

DISHNER; E. K., y READENCF, J. E.: "A systematic procedure for teaching main idea». Reading World, $1977,16,292-298$.

Doctrow, M.; WITTROCK, M. C., y MARKS, C.: «Generative processes in reading comprehension». Journal of Educational Psychology, 1978, 70, 109-118.

DONLAN, D.: "Locating main ideas in history text-books». Journal of Reading, 1980, 24, 135-140.

DUCHASTEL, P.: «Learning objectives and the organization of prose». Journal of Educational Psychology, 1979, $71,100-106$.

DuFFY, G. G.: "Teacher effectiveness research: Implications for the reading profession». En M. L. Kamil (Ed.), Directions in reading: Research and instruction, Thirtieth Yearbook of the National Reading Conference (pp. 113-136). Washington, D. C.: National Reading Conference, 1981.

DUFFY, G. G.: «Fighting off the alligators: What research in real classrooms has to say about reading instruction». Journal Reading Behavior, 1982, 14, 357-373.

DufFy, G. G., y Rofhler, L. F.: «Direct instruction of comprehension: What does it really mean»? Reading Horizons, 1982, 23, 35-40.

DUNN, B. R.; MATTHEws, S. R., y BIEGER, G.: Individual differences in the recall of lower-level textual information (Technical Report No. 150). Champaign, IL: Center for the Study of Reading, Universidad de Illinois, 1979.

DURKINN, D. D.: «Reading comprehension instruction in five basal reader series». Reading Research Quarterly, 1983, 16, 515-544.

DURKINN, D. D.: Is there a match between what elementary teachers do and what basal reader manuals recommend? (Reading Education Report No. 44). Champaign, IL: Universidad de Illinois, Center for the Study of Reading, 1983.

EAMON; D. B.: "Selection and recall of topical information in prose by better and poorer readers". Reading Research Quarterly, 1978-1979, 14, 244-257.

Fisher, C. W.; Berliner, D. C.; Filby, N. N.; Marliave, R. S.; Cahen, L. S., y Dishaw, M. M.: «Teaching behaviors, academic learning time, and student achievement: An overview». En C. Denham \& A. Liberman (Eds.), Time to learn. Washington, D. C.: National Institute of Education, 1980.

FitzGERALD, J., y SpIEgEL, D. L.: «Enhancing children's reading comprehension through instruction in narrative structurem. Journal of Reading Behavior, 1983, 15, 19-36.

GOOD, T., y BROPHY, J.: Looking in classrooms (3rd ed.). Nueva York: Harper and Row, 1984.

GuThRIE, J. T. (Ed.): Comprebension and teacbing: Research reviews. Newark, DE: International Reading Association, 1981.

HANSEN, J.: "The effects of inference training and practice on young children's reading comprehension». Reading Research Quarterly, 1981, 16, 391-417.

HANSEN, J., y PEARSON, P. D.: "An instructional study: Improving the inferential comprehension of fourth grade good and poor readers». Journal of Educational Psycbology, 1983, 79, 821-829.

Hare, V., y MiLligan, B.: «Main idea identification: Instructional explanations in four basal reader series". Journal of Reading Behavior, (en prensa).

JENKINS, J. R., y PANY, D.: «Teaching reading comprehension in the middle grades». En R. J. Spiro, B. C. Bruce, \& W. F. Brewer (Eds.), Theoretical issues in reading comprehension (pp. 555-574). Hillsdale, $N$. J.: Lawrence Erlbaum Associates, 1980.

JHONSON, R. E.: «Recall of prose as a function of structural importance of the linguistic units». Journal of Verbal Learning and Verbal Bebavior, 1970, 9, 12-20.

Johnson, D. D., y BARRET, T. C.: «Prose comprehension: A descriptive analysis of instructional practices». En C. M. Santa \& B. L. Hayes (Eds.), Children's prose comprebension: Research and practice (pp. 72 102). Newark, DE: International Reading Association, 1981.

Johnson, D. D., y PEARSON, P. D.: Teaching reading vocabulary. Nueva York: Holt, Rinehart \& Winston, 1978.

JOLLY, H. B.: «Determining main ideas: A basic study skill». En L. E. Hafner (Ed.), Improving reading in secondary schools: Selected reading (Pp. 181-190). Nueva York: Macmillan, 1967.

KINTSCH, W., y KEENAN, J. M.: «Reading rate and retention as a function of the number of propositions in the base structure of sentences». Cognitive Psychology, 1973, 5, 257-274.

Kinsch, W.; Kozminsky, E.; STreby, W. J.; MCKoON, G., y KeEnan, J. M.: «Comprehension and recall of text as a function of content variables». Journal of Verbal Learning and Verbal Bebavior, 1975, 14, 196-214.

MASON, J. M.: "An examination of reading instruction in third and fourth grades». The Reading Teacher, $1983,36,906-913$. 
MCCoNkil, G. W.: «Learning from text". Review of Researcb in Education, 1978, 5, 3-48.

MFYER, B. J. F.: The organization of prose and its effects on memory. Amsterdam: North-Holland, 1975.

MFYFR, B. J. F.: "The structure of prose: Efiects on learning and memory and inplications for educational practice». En R. C. Anderson, R. J. Spiro, \& W. E. Montagne (Eds.), Scbooling and the acquisition of knowledge (pp. 179-200). Hillsdale, N. J.: Lawrence Erlbaum Associates, 1977.

MEYER, B. J. F, y MCCONKIF, G. W.: "What is recalled after hearing a passage?" Journal of Educational Psycbology, 1973, 65, 109-117.

MOORE, D. W., y READENCE, J. E.: “Processing main ideas through parallel lesson transferm. Journal of Reading, 1980, 23, 589-593.

MOORE, D. W., y READENCE, J. E.: "A framework for reading comprehension research". Journal of Research and Development in Education, 1983, 17, 12-19.

National Assfassment of Educational Progrfas: Reading, writing, and thinking. 1979-1980 Report. Washington, D.C.: U. S. Printing Office, 1981.

Oтто, W., y Askov, E.: Rationale and guidelines: The Wisconsin design for reading skill development. Minneapolis: National Computer Systems, 1974.

OTTO, W., BARRFTT, T. C., y KOENKF, K.: «Assessment of children's statements of the main idea in reading». En J. A. Figurel (Ed.). Reading and realism (pp. 692-697). Newark, DE: International Reading Association, 1968.

Patching, W.; Kameenul, E.; Carnine, D.; Gersten, R., y Colvin, G.: «Direct instruction in critical readingskills". Reading Research Quaterly, 1983, 18, 406-418.

PEARSON, P. D.: A context for instructional research on reading comprebension. (Technical Report No. 230). Campaign. IL: University of Illinois, Center for the Study of Reading, 1982.

PEARSON, P. D.: (Ed.). Handbook of reading research. New York: Longman, 1984a.

Pearson, P. D.: «Direct explicit teaching of comprehension». En G. G. Duffy., L. R. Roehler, \& J. Mason (Eds.). Comprebension instruction: Perspectives and suggestions (pp. 222-233). Nueva York: Longman, 1984b.

Prarson, P. D., y Gallaghir, M. C.: "The instruction of reading comprehension». Contemporary Educational Psycbology, 1983, 8, 317-344.

Pearson, P. D., y Johnson, D. D.: Teaching reading comprebension. Nueva York: Holt, Rinehart \& Winston, 1978.

Prescotr, G. A.; Balow, I. H.; Hogan, T. P., y FARr, R. C.: Metropolitan achievement tests. Nueva York: The Psychological Corporation, 1978.

PutNAM, L. R.: "Don't tell them to do it... Show them how". Journal of Reading, 1974, 18, 41-43.

RAO, C. R.: Linear statistical inference and its applications (2nd ed.). Nueva York: Wiley, 1973.

RAPHAEL, T. E., y MCKINNEY, J.: «An examination of fifth-and eighth-grade children's question-answering behavior: An instructional study in metacognition". Journal of Reading Behavior, 1983, 15, 67-86.

RAPHAEL, T. E., y PEARSON, P. D.: The effect of metacognitive training on cbildren's question-answering bebavior (Technical Report No. 238). Champaign, IL: University of Illinois, Center for the Study of Reading, 1982.

REDER, L. M.: Comprebension and retention of prose: A literature review. (Technical Report No. 108). Champaign, IL: University of Illinois, Center for the Study of Reading, 1978.

Reis, R., y SpkKMAN, N. J.: "The detection of reader-based versus text-based inconsistencies and the effects of direct training of comprehension monitoring among upper-grade poor comprehenders". Journal of Reading Bebavior, 1983, 15, 49-60.

ROSENSHINE, B. V.: «Skill hierarchies in reading comprehension». En R. J. Spiro, B. C. Bruce, \& W. F. Brewer (Eds.), Theoretical issues in reading comprebension (pp. 535-554). Hillsdale, NJ: Lawrence Erlbaum Associates, 1980a.

Rosenshine, B. V.: "How time is spent in elementary classrooms». En C. Denham \& A. Lieberman (Eds.), Time to learn. Washington, DC: National Institute of Education, 1980b.

Rosenshine, B. V., y BERLINER, D. C.: «Academic engaged time». British Journal of Teacher Education, 1978, 4, 3-16.

Rosenshine, B. V., y Sthvens, R.: «Classroom instruction in reading». En P. D. Pearson (Ed.), Handbook of reading research (pp. 745-798). Nueva York: Longman, 1984.

SANTA, C., y HAYES, B. L. (Eds.): Children's prose comprehension: Research and practice. Newark, DE: International Reading Association, 1981.

SAnteusanio, R. P.: A practical approach to content area reading. Boston: Addison-Wesley, 1983.

Smiley, S. S.; OAkley, D. D.; Worthen, D.; Campione, J. C., y Brown, A. L.: «Recall of thematically relevant material by adolescent good and poor readers as a function of written versus oral presentation. Journal of Educational Psychology, 1977, 69, 381-387.

SPIRO, R. J.; BRUCE, B. C., y BREWER, W. F. (Eds.): Theoretical issues in reading comprebension: Perspectives from cognitive psychology, linguistics, artifical intelligence, and education. Hillsdale, NJ: Lawrence Erlbaum Associates, 1980.

TAYLOR, B. M.: “Children's memory for expository text after reading». Reading Research Quarterly, '1980, $15,399-411$

TAYLOR, B. M., y BFRkOWITZ, S.: «Facilitating children's comprehension of content material». En M. L. Kamil \& A. J. Moe (Eds.), Perspectives in reading researcb and instruction. Twenty-ninth Yearbook of the National Reading Conference (pp. 64-68). Clemson, SC: National Reading Conference, 1980.

TAYLOR, M. B., y Williams, J. P.: «Comprehension of learning-disabled readers: Task and text variations». Journal of Educational Psychology, 1983, 75, 743-751.

TAYLOR, M. J.: "Using photos to teach comprehension skills". Journal of Reading, 1978, 21, 514-517.

THARP, R. G.: «The effective instruction of comprehension: Results and description of the Kamehameha Early Education Program». Reading Research Quarterly, 1982, 17, 503-527. 
ThORNDYKE, P. W.: «Cognitive structures in comprehension and memory of narrative discourse». Journal of Verbal Learning and Verbal Behavior, 1977, 9, 77-110.

TIERNEY, R. J., y BRIDGe, C. A.: "The functions of inferences: An extended examination of discourse comprehension». En M. L. Kamil \& A. J. Moe (Eds.), Reading Research: Studies and applications. Twenty-eighth Yearbook of the National Reading Conference (pp. 129-133). Clemson. SC: National Reading Conference, 1979.

Tierney, R.; BRIDGe, C., y Cera, M.: "The discourse processing operations of children». Reading Researcb Quarterly, 1978-1979, 14, 539-597.

TIERnEY, R., y CunNingham, J.: «Research on teaching reading comprehension». En P. D. Pearson (Ed.), Handbook of reading research (pp. 609-655). Nueva York: Longman, 1984.

WATERS, H. S.: "Superordinate-subordinate structure in semantic memory: The roles of comprehension and retrieval processess. Journal of Verbal Learning and Verbal Bebavior, 1978, 17, 587-597.

Williams, J. P.; TAYlor, M. B., y Decani, J. S.: «Constructing macrostructure for expository text». Journal of Educational Psychology. (En prensa).

Williams, J. P.; TAYlor, M. B., y Ganger, S.: «Text variations at the level of the individual sentence and the comprehension of simple expository paragraphs". Journal of Educational Psychology, 1981, 73, 851-865.

Williams, J. P.: Skill in identification of main ideas in expository text. Paper presented at the Annual Meeting of the American Educational Research Association. Nueva Orleáns, 1984, abril.

WiNOGRAD, P. N.: "Strategic difficulties in summarizing texts». Reading Research Quarterly, 1984, 19(4), 404-425.

WINOGRAD, P. N., y BrennaN, S.: «Main ideas instruction in the basal readers». En J. A. Niles \& L. A. Harris (Eds.), Searches for meaning in reading/language processing and instruction, Thirty-second Yearbook of the national Reading Conference (pp. 80-86). Rochester, NY: National Reading Conference, 1983.

\section{NOTAS}

El autor quiere agradecer a Patrick Shannon, Edward Kameenui y a Peter Winograd por su respuesta a las versiones anteriores de este articulo. El autor aprecia enormemente la ayuda de P. David Pearson y S. Jay Samuels y de los miembros de la Editorial Advisory Board que revisaron el manuscrito. El autor también agradece a Mr. Steven Wittennauer, Principal del Frontier Elemental/Middle School, Brookston, Indiana, y a su competente cuerpo docente por su ayuda en la realización de este estudio.

1 Hay que señalar que la instrucción administrada al grupo Básico era un programa distinto del usado en el grado 6; por lo tanto, los estudiantes no estaban familiarizados con estos temas, aunque todos los alumnos del grado 6 habíaN recibido durante el año instrucción sobre las ideas principales como parte del programa del libro de lectura básico utilizado.

2 Los valores de la F en todos los MANOVA estaban basados en la lambda de Wilks que se transformó en una F aproximada, mediante la fórmula de Rao (1973).

\section{Apéndice $A$}

\section{Ejemplo de lección que emplea un modelo de instrucción directa (lección 2: ideas principales y detalles en los párrafos-implicitos).}

Intorducción: "¿Recordáis la última clase cuando aprendimos cómo encontrar las frases correctas sobre las ideas principales en los párrafos? Llamamos a esas frases frases-tema. Hoy aprenderemos a encontrar las ideas principales en los párrafos que no tienen frases-tema, es decir, los párrafos que de hecho tienen ideas principales pero no las tienen expresadas. Aprenderéis a descifrar las ideas principales no expresadas mirando los detalles del párrafo y determinando de lo que tratan todos ellos; eso será la idea principal. Esta es una destreza importante porque muchos párrafos no expresan las ideas principales y si podéis deducir cuáles son, entenderéis y recordaréis la información más importante de lo que habéis leido».

Ejemplo: «Mirad el ejemplo de esta diapositiva».

Mi padre sabe freir huevos con bacon bastante bien. También hace bollos que saben de maravilla. Cocina unas pizzas excelentes y palomitas de maiz. Sin embargo, lo que mejor le sale son las hamburguesas a la parrilla.

"Mientras yo leo el párrafo en voz alta, seguid el texto en silencio» (el profesor lee el párrafo). “Daos cuenta de que no hay una frase que establezca la idea principal, es decir, no hay una frase-tema. Además, el párrafo entero está formado por una serie de detalles.

Sin embargo, esto no quieré decir que no haya una idea principal en este párrafo. Lo que aprenderemos a hacer hoy es examinar párrafos como éste que contienen una idea principal no expresada y luego extraer dicha idea.n

Instrucción Directa: "Vamos a examinar este párrafo de la diapositiva para ver si podemos determinar su idea principab). ¿Recordáis nuestra última lección donde aprendimos a extraer el tema de un párrafo, 
es decir, la palabra o dos palabras que decian de lo que trataba el párrafo? ¿Cuál será el tema de este párrafo? ¿Sería "papa cocinando»? (respuesta de los alumnos). «Muy bien, el tema de este párrafo es "papá cocinando"." "Ahora vamos a hacer una lista en la pizarra de todas las ideas acerca de "papa cocinando". ¿quién puede ayudarnos?") (los estudiantes responden estableciendo las cuatro frases de los detalles del párrafo y el profesor las escribe en la pizarra en una lista numerada). "Muy bien. Estas son las ideas que nos informan de "papá cocinando", y ya hemos aprendido que se llaman detalles complementarios. Si estos detalles cotresponden a una misma idea principal los examinaremos para ver si podemos extraer la idea principal de este párrafon (el profesor vuelve a leer los detalles complementarios escritos en la pizarra). "¿Cuál es la idea principal que puede relacionar todos estos detalles?" (el profesor escribe las respuestas de los alumnos en la pizarra). " $S i$, hay varias maneras de decir cuál es la idea principal: "Papá sabe cocinar muchas cosas diferentes" o "Papá es un buen cocinero". Pero la idea principal nos habla de todos los detalles del párrafo; es decir, es la idea más importante del párrafo.»

"Ahora, mirad esta diapositiva. ¿Quién puede decirme qué es?" (respuesta de los estudiantes). «Si, es una mesa. Vamos a usar esta mesa para entender cómo la idea principal y los detalles van juntos. Asi como una mesa está apoyada en sus patas, también una idea principal está apoyada en los detalles. Por eso, identificamos la idea principal con la mesa y los detalles con las patas. ¿quién puede hacerlo? Vamos a empezar con las patas" (los estudiantes responden y el profesor escribe los detalles en las patas). "Ahora, pondremos la idea principal en la mesa" (el profesor escribe la idea principal en la mesa). "Así como las patas sujetan la mesa, también los detalles del párrafo sujetan la idea principal. Cuando intentéis extraer la idea principal del párrafo, pensad en la mesa y en las patas, para ayudaros a entender cómo los detalles y la idea principal se relacionan.» (El profesor luego pone un segundo ejemplo similar.)

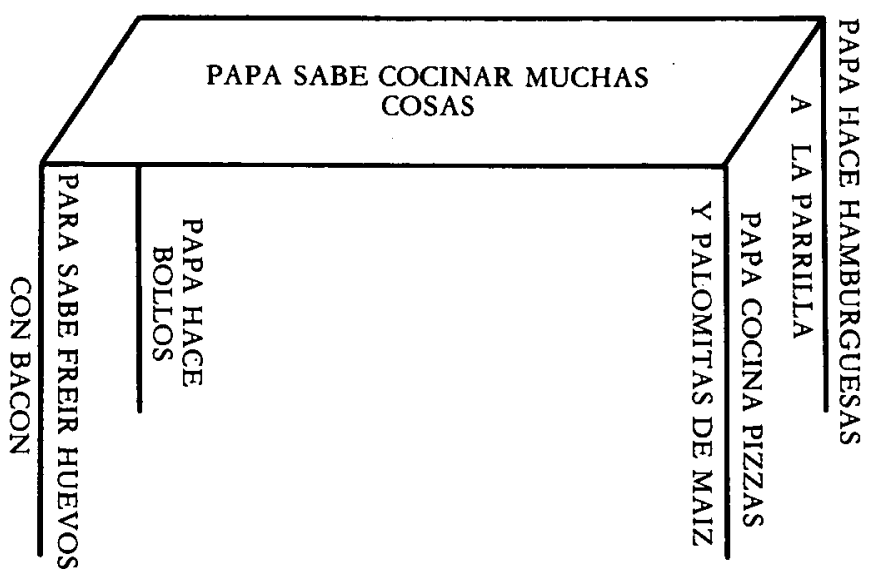

Aplicación dirigida por el profesor: "Tengo aqui unos ejemplos que debemos completar juntos.» (El profesor distribuye los ejemplos.) "Mirad el párrafo $n .{ }^{\circ} 1$. Leedlo en silencio e intentad extraer la idea principal. No hay una frase-tema en este párrafo, por eso debemos utilizar los detalles complementarios para determinar la idea principal. Señalad la letra de la frase que pensáis que mejor establece la idea principal del párrafo." (Los alumnos realizan el ejercicio.) "Muy bien, ¿qué habéis puesto? ¿Quién puede decirnos cuál es la idea principal?" (respuesta del estudiante-s). "La respuesta correcta es la "c", "los animales ayudan a las personas de diferentes maneras" es la idea principal. ¿quién puede decirnos cuáles son los detalles complementarios de dicha idea?" (respuesta de los estudiantes). «Bien, ahora hagamos el ejercicio 2. Leed el párrafo en voz baja y escribid la idea que para vosotros es la principal en la linea que hay debajo del párrafo. Pensad en el ejemplo de la mesa y las patas para ayudaros" (los estudiantes responden al ejercicio). ¿Quién puede decirnos cuál es la idea principal??" (El profesor y los alumnos continúan haciendo el resto de los ejercicios. Si los estudiantes tuvieran alguna dificutad, el profesor les ofrecerá más ejemplos).

Práctica Independiente: «Lo último que haremos en esta lección será completar unos ejercicios sin mi ayuda» (el profesor distribuye las hojas). "En esta hoja hay algunos párrafos de los que debéis extraer las ideas principales, puesto que no hay frase-tema en ninguno de ellos. En el n. 1 sólo debéis rodear la letra que corresponda a la frase que pensáis que expresa la idea principal, y en el n. 2 escribir una idea principal para cada párrafo. Cuando hayáis acabado, intentad recordar cómo se obtiene la idea principal a partir de los detalles; también podéis usar el ejemplo de la mesa para ayudaros. Trabajad con cuidado y hacedlo lo mejor posible.»

\section{APENDICE B}




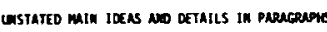
twoepenoent meactice

1. Bead the following paragraphs and circle the letter of the stutemath that best tells the min ides for each paragraph.

some dinosours were thoge aniwals that were nuch lerger than lephants. Some dinosaurs rene swall animols no larger ehap chicken. Many dinasours.

(a) Som dimosaurs were chickems.

(b) All dinosaurs ere muge, lerge minols.

(c) Oinosaurs were of different sizes.

Meny coos guard peoples: houses and stores. Some dogs are seelng-eye cogs and help blind people wive inound sately. Some dogs with noses art used to hion.

(a) cogs help blind poople.
(b) Dogs help people in different was.
(c) Dogs ore yood friends.

(c) Dogs ine good friends.

Manu seople are gware that the giraffe is the tollest anter l on earth, but fer realize chat despite its long neck, the giraffe has the sase nower of neck bones that howns nave-only seven. Also, many people giraffes gartiy use their vice, they have rocal cords and are quit to copable of muling noises then they choose to. The homs- that grow on thetr heads art not really noms at all. Insteat. thay

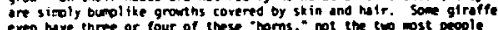

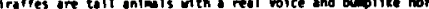

(b) Giraffes can mike sounds, even though people think they cansot.

(d) Giraffes and humans are alike in several igs.

2. Iech of the following poregrephs heve unstated win ideas. Read each

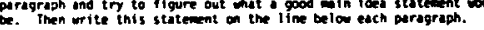

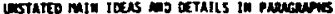

\section{$\operatorname{cosentans}$}

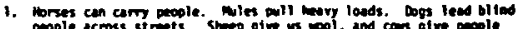
people across

(b) Dorses qive us wy different foods to wat.

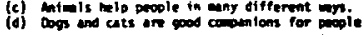

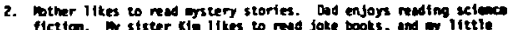

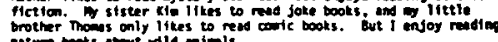

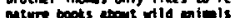

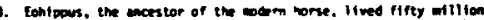

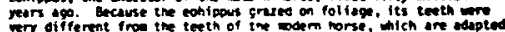

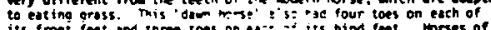
its front leet and inree coes on ta: : " Its nino feet. rorses of todiy. mowerer. nat

4. Hern treasure mating began in the rorthestem unted states in the late 1960s. Todsy, mearly one rit i ion people particisate in

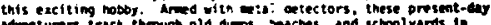

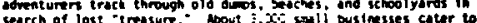

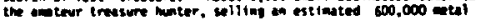
tetectors eech ger.

yy wother wre bedroow sllpoen ehis worning. Then she put on nowing

shoes for Jogging. After Jogping. she put on her old shoes to wort the garcen. Alter linen, she put on her ne- sne thers to play tents. dimer.

Duppins, of course, can be mede into ples. Out poptins can siso used to mike soup and cakt. Some people roast puppolin seeds and eat 等 thellionet.

A person with a metsl betector will manlly find things like bottle caps.

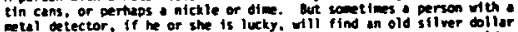

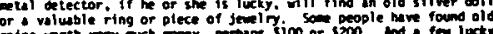

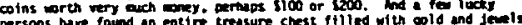

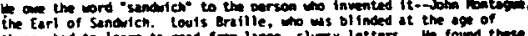
three, had to leam to resd froe lorge, cluesy letters.

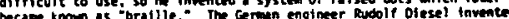

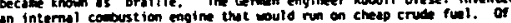

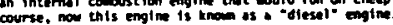




\section{Apéndice B}

Medidas dependientes usadas para determinar la comprensión de las ideas principales y de los detalles complementarios

Porion of Main Ideas in Paragraphs Test

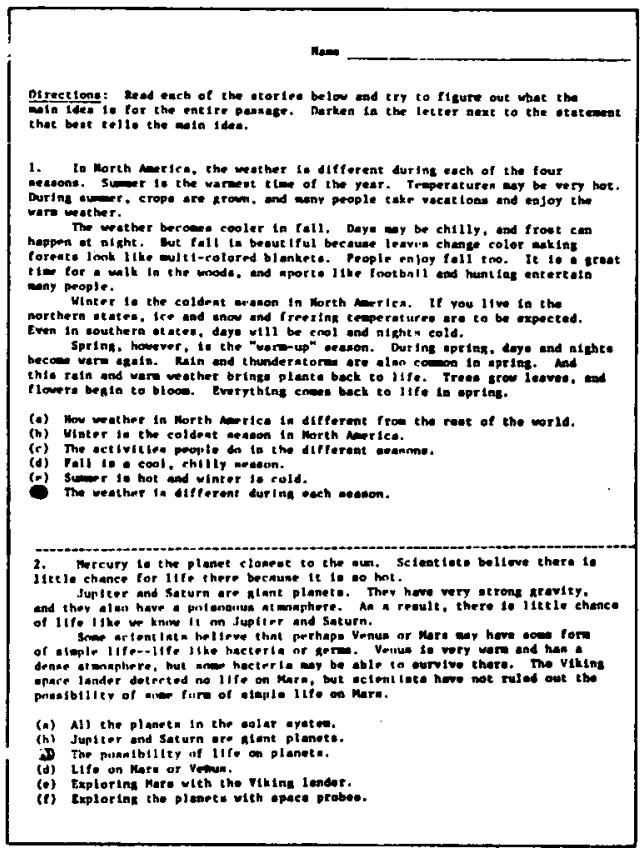

Portion of

Details that Support Main Ideas Test

\begin{tabular}{|c|c|}
\hline \multicolumn{2}{|r|}{ 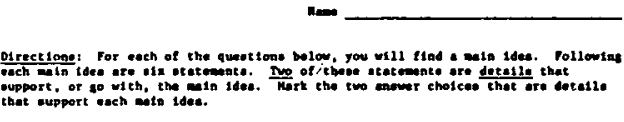 } \\
\hline $\begin{array}{l}\text { Practice } \\
\text { (a) } \\
\text { (c) } \\
\text { (d) } \\
\text { (I) }\end{array}$ & 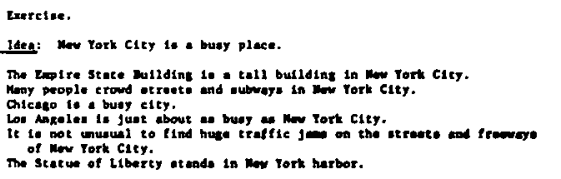 \\
\hline 1. & 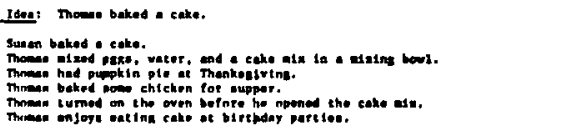 \\
\hline $\begin{array}{l}\text { Maln } \\
\text { (c) } \\
\text { (c) }\end{array}$ & 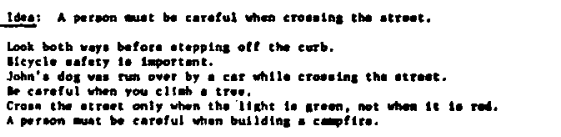 \\
\hline 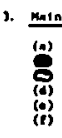 & 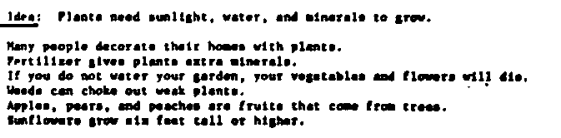 \\
\hline
\end{tabular}




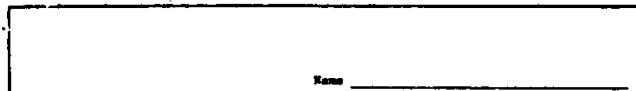

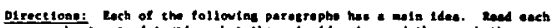

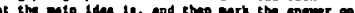

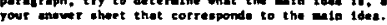

wets

Roblan bulid mats In erees. Rogleo build naste in high rocky places.

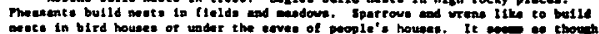

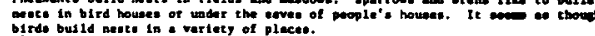

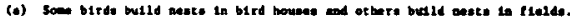

(b) Notion bulld aeste in trees.

- Brds butld meats in e vartety of pleces.

(d) tn epring. birds lay ease to their neate and later young birda hateb froe the eat:.

(a) Eales build weste in hleth rocky places.

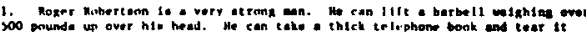

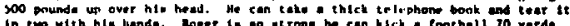

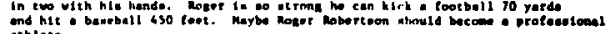
hlote.

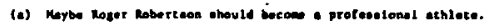

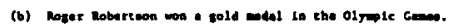

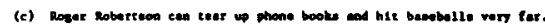

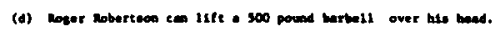

- Noper Mobortion to a wry otrons nom.

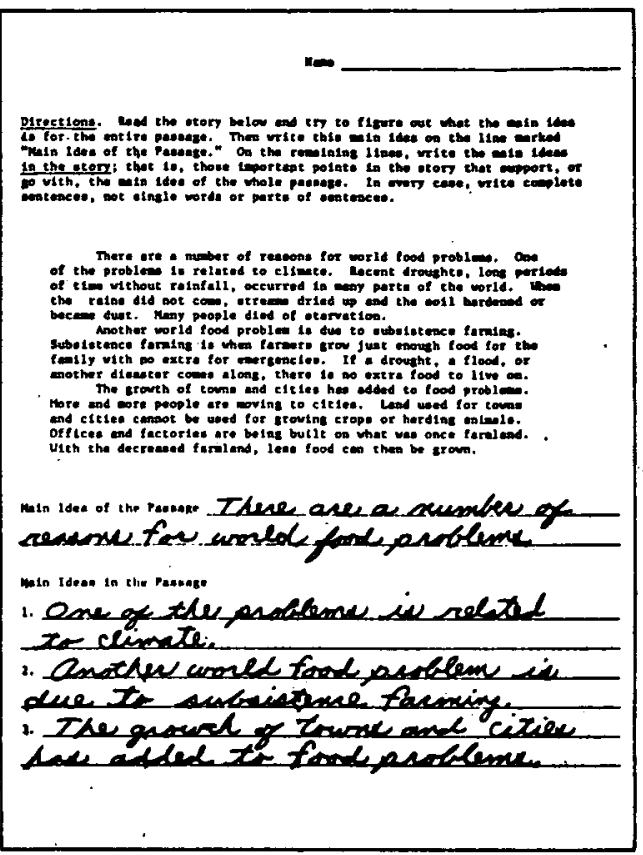

Main Idea Outline Test (Implicit)

$+2 \cdot$

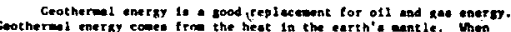

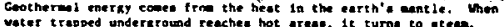

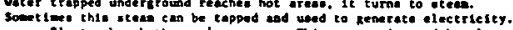

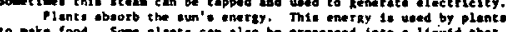
to make food. Som plent, ces aleo be processed inte I I puid thet

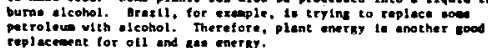

stplacemat for oll and all merky. If oll vinds blew at apped of 21 alles per hnus, che enerey

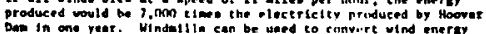

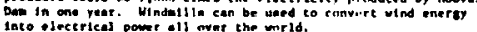

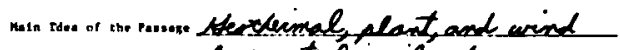

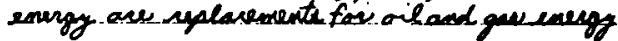
wain iluen in thie manaser

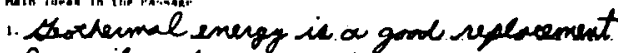
for ail and gad energy.

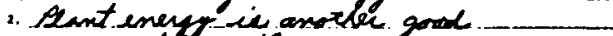

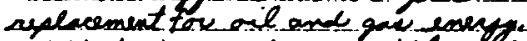

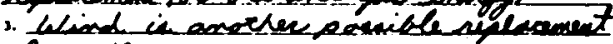
terecil and gan enesy
Free Recall Test Passage

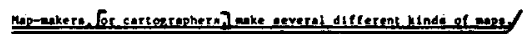

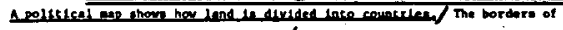

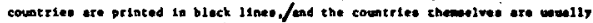

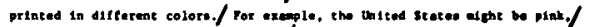

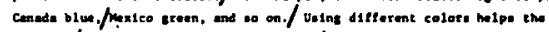

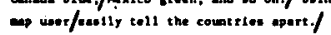

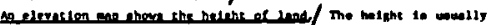

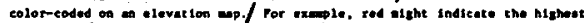

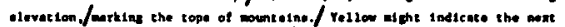

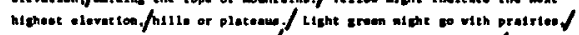

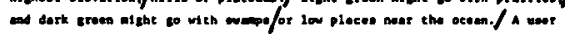

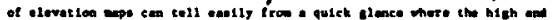
10u parce of leme areas arr./

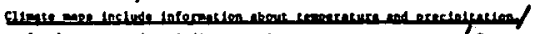

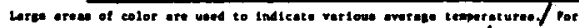

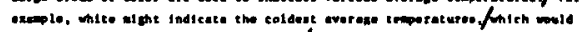

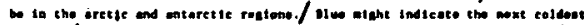

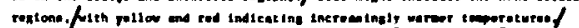

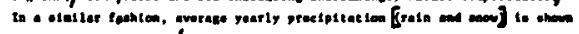

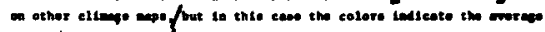

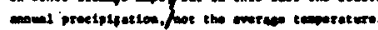

Nole. Alternate correct responses fot the passage main idea were:

- The replacements for oil and ass energy.

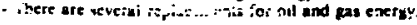

